\title{
Phase Ib/II Study of Capmatinib (INC280) Plus Gefitinib After Failure of Epidermal Growth Factor Receptor (EGFR) Inhibitor Therapy in Patients With EGFR-Mutated, MET Factor-Dysregulated Non-Small-Cell Lung Cancer
}

Yi-Long Wu, Li Zhang, Dong-Wan Kim, Xiaoqing Liu, Dae Ho Lee, James Chih-Hsin Yang, Myung-Ju Ahn, Johan F. Vansteenkiste, Wu-Chou Su, Enriqueta Felip, Vincent Chia, Sabine Glaser, Philippe Pultar, Sylvia Zhao, Bin Peng, Mikhail Akimov, and Daniel S.W. Tan

Author affiliations and support information (if applicable) appear at the end of this article.

Published at jco.org on August 29, 2018. Clinical trial information: NCT01610336.

Corresponding author: Yi-Long Wu, MD, Guangdong Lung Cancer Institute,

Guangdong General Hospital, and Guangdong Academy of Medical Sciences, 106 Zhongshan Er Rd, Guangzhou 510080, People's Republic of China; e-mail: syylwu@live.cn.

(C) 2018 by American Society of Clinical Oncology

0732-183X/18/3699-1/\$20.00
ASSOCIATED CONTENT

(2) Appendix

DOI: https://doi.org/10.1200/JCO 2018.77.7326

DOI: https://doi.org/10.1200/JCO.2018. 77.7326

\section{$\begin{array}{llllllll}\text { A } & \text { B } & \text { S } & \text { T } & \text { R } & \text { A } & \text { C } & \text { T }\end{array}$}

\section{Purpose}

Mesenchymal-epithelial transition factor (MET) dysregulation occurs in up to $26 \%$ of non-small-cell lung cancers (NSCLCs) after epidermal growth factor receptor (EGFR)-tyrosine kinase inhibitor (TKI) treatment. Capmatinib (INC280) is a potent and selective MET inhibitor with preclinical activity in combination with gefitinib in EGFR-mutant, MET-amplified/overexpressing models of acquired EGFR-TKI resistance. This phase $\mathrm{lb} / \mathrm{ll}$ study investigated the safety and efficacy of capmatinib plus gefitinib in patients with EGFR-mutated, MET-dysregulated (amplified/overexpressing) NSCLC who experienced disease progression while receiving EGFR-TKI treatment.

\section{Methods}

Patients in phase Ib received capmatinib 100- to 800-mg capsules once per day or 200- to 600-mg capsules or tablets twice per day, plus gefitinib $250 \mathrm{mg}$ once per day. Patients in phase II received the recommended phase II dose. The primary end point was the overall response rate (ORR) per Response Evaluation Criteria in Solid Tumors (RECIST) version 1.1.

\section{Results}

Sixty-one patients were treated in phase $\mathrm{Ib}$, and 100 were treated in phase II. The recommended phase II dose was capmatinib 400 mg twice per day plus gefitinib 250 mg once per day. Preliminary clinical activity was observed, with an ORR across phase Ib/II of $27 \%$. Increased activity was seen in patients with high MET-amplified tumors, with a phase II ORR of $47 \%$ in patients with a METgene copy number $\geq 6$. Across phases $\mathrm{lb}$ and $\mathrm{II}$, the most common drug-related adverse events were nausea (28\%), peripheral edema (22\%), decreased appetite (21\%), and rash $(20 \%)$; the most common drug-related grade $3 / 4$ adverse events were increased amylase and lipase levels (both 6\%). No significant drug-drug interactions between capmatinib and gefitinib were evident.

\section{Conclusion}

This study, focused on a predominant EGFR-TKI resistance mechanism in patients with EGFRmutated NSCLC, shows that the combination of capmatinib with gefitinib is a promising treatment for patients with EGFR-mutated, MET-dysregulated NSCLC, particularly MET-amplified disease.

\section{J Clin Oncol 36. (C) 2018 by American Society of Clinical Oncology}

\section{INTRODUCTION}

Dysregulation of the MET proto-oncogene receptor tyrosine kinase frequently occurs as a resistance mechanism to epidermal growth factor receptor (EGFR)-tyrosine kinase inhibitor (TKI) therapy. Patients with EGFR-mutated non-small-cell lung cancer (NSCLC) usually relapse within a year, despite high response rates to EGFR-TKIs. ${ }^{1}$ MET dysregulation has been implicated as a therapeutically tractable resistance mechanism in a significant number of these patients, with $M E T$ amplification (activating ERBB3 signaling ${ }^{2}$ ) reported in $5 \%$ to $26 \%$ of NSCLCs with EGFR inhibitor resistance. ${ }^{2-7}$

Capmatinib (INC280) is a highly specific and potent MET inhibitor in biochemical and cellular assays that causes regression of MET-dependent (amplified/autocrine) tumors in animal models at well-tolerated doses. ${ }^{8}$ Single-agent activity has been observed against EGFR wild-type tumor 
models with strong MET amplification, ${ }^{9}$ mutation, ${ }^{10}$ and/or overexpression. ${ }^{9}$ Capmatinib has also demonstrated preclinical activity in EGFR-mutant, MET-activated NSCLC when combined with first-generation ${ }^{9}$ and third-generation (unpublished data) EGFR-TKIs. For example, the combination of capmatinib and gefitinib is active in EGFR-mutant/MET-amplified models of acquired EGFR inhibitor resistance. ${ }^{9}$ Furthermore, capmatinib restores sensitivity to erlotinib and promotes apoptosis in NSCLC models rendered erlotinib-resistant by hepatocyte growth factor. ${ }^{11}$

Here, we report the results from a phase Ib/II study investigating the safety and efficacy of capmatinib in combination with gefitinib in patients with EGFR-mutated, MET-dysregulated (METamplified or MET-overexpressing) NSCLC who had experienced disease progression while receiving EGFR-TKI treatment.

\section{METHODS}

\section{Study Oversight}

This study was performed in accordance with the Declaration of Helsinki and the principles of Good Clinical Practice. The protocol was approved by the institutional review boards at each investigative site, and all patients provided written informed consent before any study procedures. The study was designed by the sponsor (Novartis Pharma AG, Basel, Switzerland).

\section{Study Design}

The phase Ib part of the study used an adaptive five-parameter Bayesian logistic regression model (BLRM) ${ }^{12}$ guided by escalation with overdose control to determine the maximum tolerated dose (MTD) on the basis of dose-limiting toxicities (DLTs) recorded in cycle 1 . With the introduction of a twice-per-day dosing schedule in addition to a once-perday schedule, a second BLRM model was fitted with prior information on the basis of the once-per-day information. A tablet formulation was introduced after the capsule formulation to improve patient compliance and convenience, and an additional BLRM was used to assess safety and guide dose recommendations. The prior distributions for this model incorporated existing dose-toxicity data for single-agent capmatinib/gefitinib and the combination.

The primary objective was to determine the MTD and/or recommended phase II dose (RP2D) in phase Ib, and to estimate the overall response rate (ORR; per Response Evaluation Criteria in Solid Tumors [RECIST] version 1.1) to capmatinib in combination with gefitinib in patients with MET-dysregulated NSCLC in phase II. Secondary objectives were to estimate time-dependent clinical activity of capmatinib plus gefitinib (overall survival, duration of response, and progression-free survival $[\mathrm{PFS}]$ ), determine the safety and tolerability of capmatinib plus gefitinib, and characterize the pharmacokinetic (PK) profile. An exploratory objective was to study the pharmacodynamics (PD) of capmatinib plus gefitinib.

\section{Patients}

Overall eligibility criteria were age $\geq 18$ years; Eastern Cooperative Oncology Group performance score $\leq$ 2; EGFR-mutant NSCLC (exon 19 deletion or L858R); acquired resistance to gefitinib, erlotinib, or afatinib treatment according to published acquired resistance criteria (documented clinical benefit as per RECIST and demonstrated progression while receiving continuous treatment or $\leq 30$ days since EGFR-TKI administration $)^{13}$; and demonstration of MET dysregulation. For phase Ib, patients were required to have $M E T$-amplified tumors, defined as either $M E T$ gene copy number $(\mathrm{GCN}) \geq 5$ and/or a $M E T /$ centromere ratio of $\geq 2.0$, or MET overexpression, defined as $\geq 50 \%$ of tumor cells with moderate or strong staining intensity. For phase II, patients were required to have experienced RECIST-recorded clinical benefit while taking a prior singleagent EGFR-TKI before progression. They were also required to be METdysregulated after disease progression while receiving an EGFR-TKI, which was initially defined as $M E T$ GCN $\geq 5$ as determined by fluorescence in situ hybridization (FISH) or $50 \%$ of tumor cells with immunohistochemistry (IHC) $2+/ 3+$, determined locally or centrally. In subsequent protocol amendments, these criteria were revised to $50 \%$ of tumor cells with IHC $3+$ or IHC $2+$ plus MET GCN $\geq 5$ and then to $50 \%$ of tumor cells with IHC $3+$ or $M E T$ GCN $\geq 4$, determined centrally.

Patients with a known or documented EGFR T790M mutation were initially allowed (five patients, assessed centrally) but were later excluded through a protocol amendment. Other exclusion criteria included previous treatment with a MET inhibitor or hepatocyte growth factor-targeting therapy, receipt of more than two lines of chemotherapy and more than one line of EGFR-TKI therapy (gefitinib, erlotinib, or afatinib), and the presence of symptomatic CNS metastases that were neurologically unstable or required increasing doses of steroids.

\section{Treatment Plan and Drug Administration}

In phase $\mathrm{Ib}$, patients were treated with gefitinib $250 \mathrm{mg}$ once per day plus capmatinib capsules of either 100 to $800 \mathrm{mg}$ once per day or 200 to $600 \mathrm{mg}$ twice per day. The capmatinib tablet formulation was tested at 200 and $400 \mathrm{mg}$ twice per day. In the phase II expansion, patients were treated with capmatinib at the RP2D of $400 \mathrm{mg}$ twice per day (capsules or tablets) plus gefitinib $250 \mathrm{mg}$ once per day. Full treatment and drug administration details together with statistical analysis of the primary objective (phase II) are provided in the Appendix (online only).

\section{PK Analysis}

Capmatinib and gefitinib concentrations were measured in plasma using liquid chromatography-tandem mass spectrometry. Noncompartmental PK analysis was performed to generate PK parameters of capmatinib and gefitinib, and the dose proportionality of capmatinib was assessed.

\section{PD Biomarker Assessments}

Paired pretreatment and post-treatment fresh tumor (and/or available archival tissue after disease progression while receiving an EGFR-TKI) samples were collected and evaluated for PD modulation of downstream components of the MET and EGFR signaling pathways. This exploratory analysis assessed key changes in the activation of downstream markers, including phosphorylated ( $p$ )-MET, $p$-ERK, $p$-AKT, and $p$-S6 (by IHC), to determine the level of pathway inhibition induced by the capmatinib and gefitinib combination. Potential correlative MET alteration markers of treatment efficacy were also evaluated; these included $M E T$ amplification (GCN as determined by FISH) and/or protein expression (as measured by IHC). Next-generation sequencing (Foundation Medicine, Cambridge, MA) was performed where tumor tissue was available, and any MET mutations were documented.

\section{RESULTS}

At the primary analysis cutoff date of June 10, 2016, enrollment was complete; 61 patients were enrolled in the phase Ib doseescalation part, and 100 patients were enrolled in the phase II expansion (Table 1) from a total of 681 patients screened (Appendix Fig A1, online only). In phase Ib, gefitinib $250 \mathrm{mg}$ once per day plus capmatinib capsules were evaluated at the following doses: $100 \mathrm{mg}$ once per day $(\mathrm{n}=5) ; 200 \mathrm{mg}$ once per day $(\mathrm{n}=7) ; 400 \mathrm{mg}$ once per day $(n=6) ; 800$ mg once per day $(n=7) ; 200$ mg twice per day $(\mathrm{n}=4) ; 400 \mathrm{mg}$ twice per day $(\mathrm{n}=12)$; and $600 \mathrm{mg}$ twice per day $(\mathrm{n}=5)$. Capmatinib in tablet formulation was evaluated at 


\begin{tabular}{|c|c|c|c|}
\hline Characteristic & Phase lb $(n=61)$ & Phase II $(n=100)$ & All $(\mathrm{N}=161)$ \\
\hline Median age (years) & 58.0 & 61.0 & 60.0 \\
\hline \multicolumn{4}{|l|}{ Age group (years) } \\
\hline$<65$ & $46(75)$ & $62(62)$ & $108(67)$ \\
\hline$\geq 65$ & $15(25)$ & $38(38)$ & 53 (33) \\
\hline Male & $25(41)$ & $48(48)$ & $73(45)$ \\
\hline \multicolumn{4}{|l|}{ Race } \\
\hline Asian & $53(87)$ & $78(78)$ & $131(81)$ \\
\hline White & $8(13)$ & $22(22)$ & $30(19)$ \\
\hline \multicolumn{4}{|l|}{ ECOG performance status } \\
\hline 0 & $12(20)$ & $17(17)$ & $29(18)$ \\
\hline 1 & $45(74)$ & $81(81)$ & $126(78)$ \\
\hline 2 & $4(7)$ & $2(2)$ & $6(4)$ \\
\hline \multicolumn{4}{|l|}{ Histology } \\
\hline Adenocarcinoma & $59(97)$ & 95 (95) & $154(96)$ \\
\hline Adenosquamous cell carcinoma & 0 & $2(2)$ & $2(1)$ \\
\hline Squamous cell carcinoma & 0 & $2(2)$ & $2(1)$ \\
\hline Large cell carcinoma & $1(2)$ & 0 & $1(1)$ \\
\hline Other & $1(2)$ & $1(1)$ & $2(1)$ \\
\hline EGFR mutation status & $n=15$ & $n=47$ & $\mathrm{n}=62$ \\
\hline L858R & $12(80)$ & $20(43)$ & $32(52)$ \\
\hline Exon 19 deletion & $2(13)$ & $21(45)$ & $23(37)$ \\
\hline L858R + T790M & $0(0)$ & $5(11)$ & $5(8)$ \\
\hline G719S/A/C & $0(0)$ & $1(2)$ & $1(2)$ \\
\hline S768I & $1(7)$ & $0(0)$ & $1(2)$ \\
\hline \multicolumn{4}{|l|}{ Prior lines of therapy } \\
\hline One & $27(44)$ & $59(59)$ & $86(53)$ \\
\hline Two or more & $34(56)$ & $41(41)$ & $75(47)$ \\
\hline Prior EGFR-TKI & $61(100)$ & $100(100)$ & $161(100)$ \\
\hline \multicolumn{4}{|l|}{ EGFR-TKI as last prior therapy } \\
\hline Yes & $43(70)$ & $84(84)$ & $127(79)$ \\
\hline Gefitinib & $28(46)$ & $44(44)$ & $72(45)$ \\
\hline Erlotinib & $14(23)$ & 34 (34) & $48(30)$ \\
\hline Afatinib & $1(2)$ & $5(5)$ & $6(4)$ \\
\hline Icotinib & 0 & $1(1)$ & $2(1)$ \\
\hline No & $18(30)$ & $16(16)$ & $34(21)$ \\
\hline \multicolumn{4}{|l|}{ Tumor MET status } \\
\hline $\mathrm{GCN}<4$ & ND & $41(41)$ & - \\
\hline $4 \leq \mathrm{GCN}<6$ & ND & $18(18)$ & - \\
\hline $\mathrm{GCN} \geq 6$ & ND & $36(36)$ & - \\
\hline $\mathrm{IHCO}$ & ND & $4(4)$ & - \\
\hline $\mathrm{IHC} 1+$ & ND & $2(2)$ & - \\
\hline $\mathrm{IHC} 2+$ & ND & $16(16)$ & - \\
\hline IHC 3+ & ND & 78 (78) & - \\
\hline
\end{tabular}

$200 \mathrm{mg}$ twice per day $(\mathrm{n}=7)$ and $400 \mathrm{mg}$ twice per day (subsequently declared as the RP2D; $n=8$ ). The median duration of treatment exposure was 21.0 weeks (range, 1.0 to 975.0 weeks) from the start to the last treatment, per the data cutoff date. The overall median actual dose intensity of capmatinib was $791.6 \mathrm{mg} /$ day (mean, $662.0 \mathrm{mg} /$ day). In the 61 patients in phase $\mathrm{Ib}$, the median and mean actual dose intensities of capmatinib (all doses) were 480.0 and $556.1 \mathrm{mg} /$ day, respectively. In the 100 patients in phase II (with a planned dose of capmatinib $400 \mathrm{mg}$ twice per day plus gefitinib $250 \mathrm{mg}$ once per day), the median and mean actual dose intensities of capmatinib (tablet or capsule) were 796.3 and $726.6 \mathrm{mg} /$ day, respectively. The median duration of follow-up was 12.2 months (from enrollment to last reported follow-up at the data cutoff date). At the time of data cutoff in phase Ib, 56 of
61 patients $(92 \%)$ had discontinued treatment, most commonly because of disease progression (43 of 61 patients [70\%]); five of 61 patients $(8 \%)$ discontinued because of adverse events (AEs). At the time of data cutoff in phase II, 88 of 100 patients $(88 \%)$ had discontinued treatment, most commonly because of disease progression (69 of 100 patients [69\%]); 13 of 100 patients $(13 \%)$ discontinued because of AEs.

\section{Efficacy}

Phase $\mathrm{Ib}$ efficacy. In phase Ib $(\mathrm{n}=61)$, the ORR was $23 \%$ across all doses and regardless of MET status (Table 2). Four of eight evaluable patients treated at the RP2D of capmatinib $400 \mathrm{mg}$ twice per day (tablets) plus gefitinib $250 \mathrm{mg}$ once per day experienced a partial response (ORR, 50\%); of these four responders, 


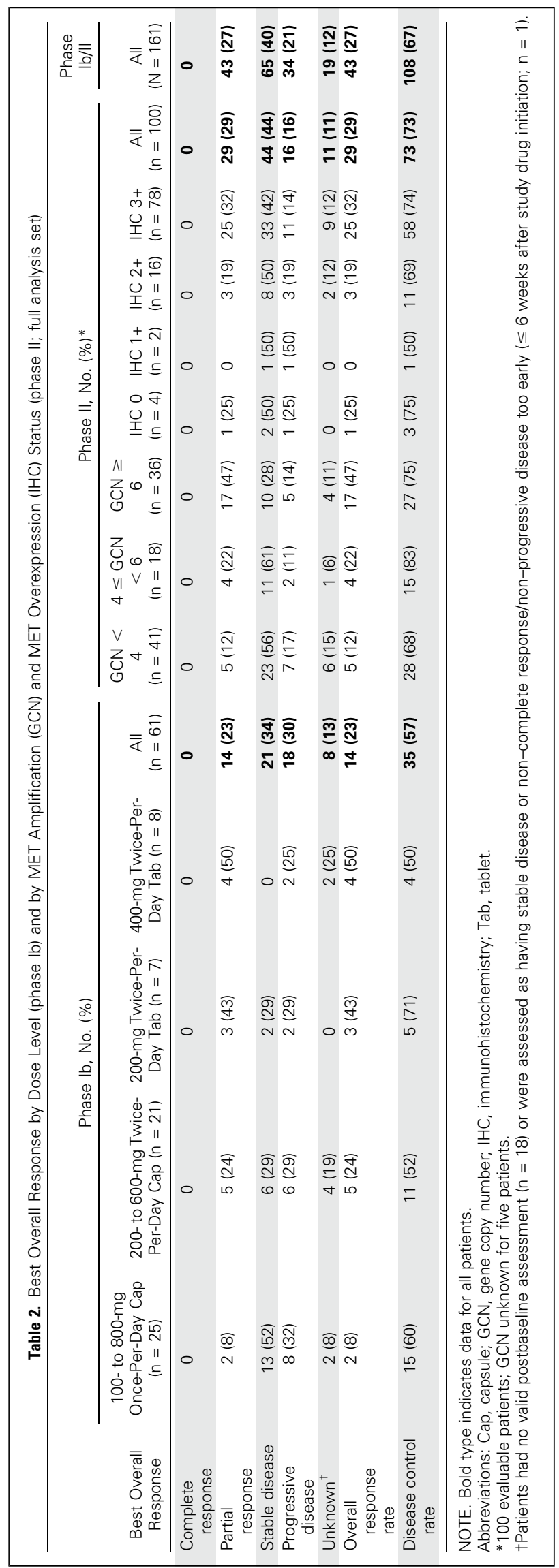


three had received an EGFR-TKI as their last prior therapy. The disease control rate (complete response, partial response, or stable disease) among all patients in phase Ib was 57\% (Table 2).

Phase II efficacy. The ORR (primary end point; investigator assessment) was 29\% (29 of 100 patients) regardless of MET status; of these responders, 25 of 29 (86\%) had received an EGFRTKI as their last prior therapy. The disease control rate was $73 \%$; median duration of response was 5.6 months (95\% CI, 3.8 to 7.2 months). In a subgroup analysis by MET GCN category, the best observed ORR was $47 \%$ in patients $(\mathrm{n}=36)$ with $M E T$ $\mathrm{GCN} \geq 6$ tumors. In the MET overexpression status IHC 3+ subgroup, the ORR was $32 \%$ (25 of 78 patients; Table 2). A similar ORR of $31.8 \%$ (27 of 85 patients) was observed in the subgroup of patients with tumors of either IHC $3+$ or IHC $2+$ plus $\mathrm{GCN} \geq 5$.

Tumor reductions for individual patients by GCN and IHC subgroup, as best percentage change from baseline in the sum of tumor lesion diameters, are presented in Figure 1. Baseline tumor molecular status (FISH GCN) by treatment group and according to IHC protein expression status are listed in Table 1 and Appendix Table A1 (online only). Median PFS in the GCN $\geq 6(n=36)$ and IHC $3+(\mathrm{n}=78)$ subgroups was 5.49 months $(95 \% \mathrm{CI}, 4.21$ to 7.29 months) and 5.45 months (95\% CI, 3.71 to 7.10 months), respectively; full PFS data are provided in the Appendix.

\section{Safety}

One of five DLT-evaluable patients treated with 800-mg onceper-day capsules experienced a DLT of grade 3 dizziness. The single evaluable patient treated with 600-mg twice-per-day capsules experienced two DLTs of grade 4 cough and grade 4 dyspnea. There were no DLTs reported in 10 patients treated with capsules or the seven patients treated with tablets at the 400-mg twice-per-day dose level. The RP2D was declared as capmatinib tablet $400 \mathrm{mg}$ twice per day plus gefitinib $250 \mathrm{mg}$ once per day.

All-grade and grade 3/4 AEs, regardless of study drug relationship, are listed in Table 3. A total of 140 of 161 patients $(87 \%)$ reported at least one $\mathrm{AE}$ believed to be related to study treatment, most frequently ( $\geq 20 \%$ of patients) nausea $(28 \%)$, peripheral edema (22\%), decreased appetite (21\%), and rash (20\%). Grade 3/4 AEs believed to be study drug related were reported in 46 of 161 patients $(29 \%)$; the most common ( $\geq 5 \%$ of patients) were increased amylase and lipase levels (both 6\%). Serious AEs were reported in 53 of 161 patients (33\%) overall, with 11 of 161 (7\%) believed to be study drug related. Overall, 27 of 161 patients (17\%) reported AEs that led to study drug discontinuation, and 71 of 161 patients (44\%) reported AEs requiring dose adjustment or interruption. Analysis of the capsule- and tablet-treated subgroups in phase II revealed that slightly fewer patients in the capsule group experienced AEs $(96 \% v 100 \%)$. A total of 85 of 161 patients $(53 \%)$ died (47 patients in phase II, of whom 34 were in the capsule group and 13 were in the tablet group); 13 of 161 patients $(8 \%)$ died during the study (up to 30 days after the end of treatment), primarily (10 of $13[77 \%]$ ) as a result of the study indication. One patient each $(<1 \%)$ died as a result of dyspnea, myocardial infarction, and pneumonia-of these, only the dyspnea was believed to be related to the study treatment; in this patient, neither pneumonitis nor infection were diagnosed. Dyspnea occurred in only $2 \%$ of patients overall, with no instances of dyspnea reported in phase II.

\section{PK Analysis}

Capmatinib was rapidly absorbed after oral administration with gefitinib, with the tablet formulation providing higher mean exposures than the capsule at the same dose levels tested. Full PK results are listed in Appendix Table A2 (online only). The mean plasma concentration versus time profiles on cycle 1 day 15 (C1D15) for the capmatinib twice-per-day regimens by dose level and formulation are provided in Figure 2. No significant drug-drug interactions between capmatinib and gefitinib were observed. Compared with single-agent capmatinib (RP2D), mean steadystate exposure (area under the plasma concentration-time curve ranging from 0 to 12 hours and maximum plasma concentration) was higher (1.34-fold and 1.30-fold, respectively) with gefitinib coadministration (unpublished data). Mean plasma exposures to gefitinib were comparable among treatments at various doses with different dose regimens or formulations of capmatinib and were in the range of exposures reported for single-agent treatment. ${ }^{14}$

\section{PD Analysis}

Exploratory analyses of baseline and C1D15 tumor biopsy MET H-scores were performed with samples from five patients treated with 400-mg twice-per-day capsules (phase Ib/II patients with noncompulsory screening/C1D15 p-MET staining intensity [H-score] data). Significant MET pathway inhibition was induced in these patients, with p-MET H-score reductions of 15 to 260 in four of the five patients (Fig 3).

\section{DISCUSSION}

The RP2D for capmatinib in combination with gefitinib $250 \mathrm{mg}$ once per day was declared as 400-mg twice-per-day tablets on the basis of a combination of safety, PK, and PD data. Preliminary clinical activity was observed in patients treated with the combination, with an ORR of $27 \%$ for all patients across phase Ib and II, and an ORR of $29 \%$ for phase II patients treated at the $\mathrm{RP} 2 \mathrm{D}$. In phase II, $86 \%$ of responding patients had received an EGFR-TKI as their last prior therapy; therefore, few patients had received intervening chemotherapy, and potential retreatment effects were not considered to affect the ORR. Notable activity was seen in patients with high MET-amplified tumors. In a post hoc subgroup analysis by MET GCN category, the best observed ORR was $47 \%$ in phase II patients with MET GCN $\geq 6$ tumors; the ORR was $32 \%$ in patients with IHC $3+$ tumors, which was comparable with that observed in patients with IHC $2+$ plus GCN $\geq 5$ tumors. Thus IHC 3+ status was predictive of response, whereas IHC 0 to $2+$ was not predictive unless combined with amplification status, although patient numbers in the IHC 0 to $1+$ categories were small. Additional studies are required to provide conclusive validation of IHC-measured MET expression as a predictive biomarker. Of note, expression of MET may not always accurately reflect MET receptor activation. ${ }^{15,16}$ However, it is yet to be established whether activated p-MET may be a more accurate indicator of MET activation than total MET expression by IHC. Overall, the exploratory 


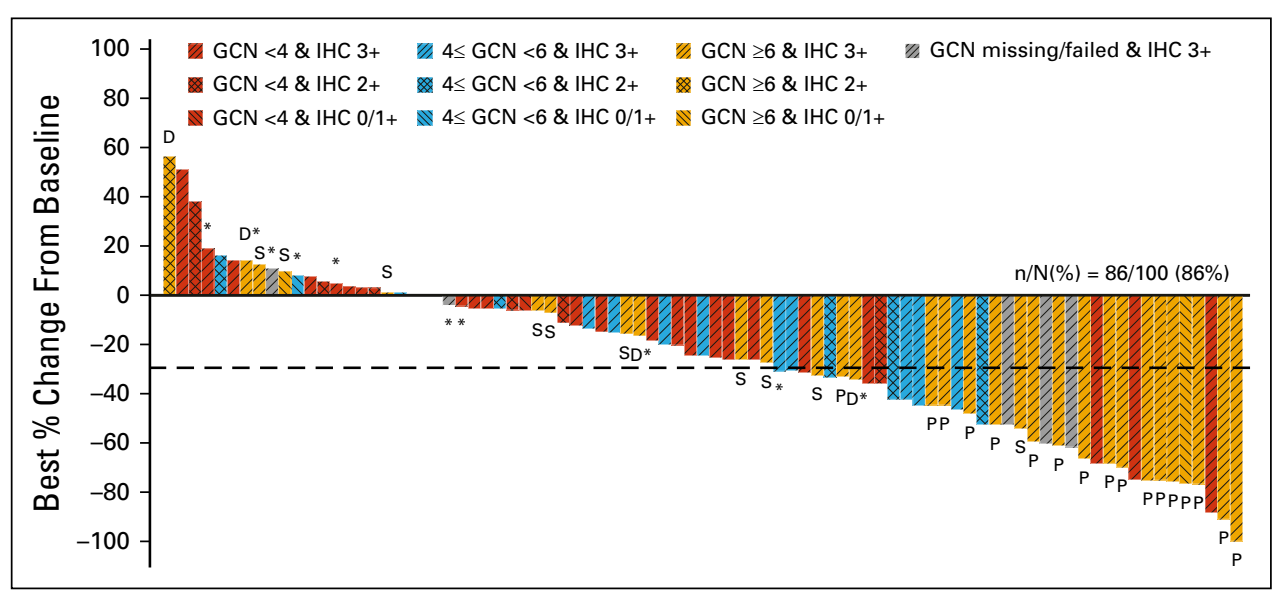

Fig 1. Best percentage change in sum of diameters of target lesions in all phase $\|$ patients by gene copy number (GCN) and immunohistochemistry $(\mathrm{IHC})$ subgroup (full analysis set). $\mathrm{N}$ represents the number of patients with a baseline and at least one post-baseline assessment of tumor lesions (investigator assessment). D, progression of disease; $P$, partial response; $S$, stable disease. $(*)$ Percentage change in sum of diameters of target lesion contradicted by overall lesion response of progressive disease.

biomarker data generated in this study indicate that MET detection by FISH using a cutoff value of GCN $\geq 6$, compared with MET staining by IHC, more accurately identifies the patient population most likely to respond to the combination of capmatinib plus gefitinib.

Although MET GCN was used to define MET positivity in this study, other FISH-based methods have been used. MET/CEP7 ratio can be used to distinguish true amplification and polysomy, ${ }^{17}$ and a ratio of $\geq 2$ is considered an equivalent positive threshold. ${ }^{16}$ However, in initial analyses of patient samples in this study (unpublished data), responses better correlated with GCN versus $M E T / C E P 7$ ratio, and on the basis of these data, GCN was selected as the biomarker.

Other recent advanced-stage studies employing different biomarker selection criteria have not provided positive data. A phase II study of the MET-directed monoclonal antibody onartuzumab plus erlotinib showed improved outcomes in patients who were MET positive by IHC, but a subsequent phase III study was negative. ${ }^{18,19}$ A phase III study of tivantinib was stopped early because of an increased incidence of interstitial lung disease; the study also failed to demonstrate any improvement in PFS or overall survival. ${ }^{20}$ However, alternative mechanisms of action have been suggested for tivantinib. ${ }^{21}$ A study of tepotinib plus gefitinib in patients who experienced disease progression while receiving EGFR-TKI therapy provided an ORR of $28 \%$ for patients with IHC $3+$ tumors, and the data suggested an increased likelihood of disease stabilization in patients with IHC $2+$ tumors. $^{22}$ A subsequent phase II study may shed more light on the utility of IHC as a biomarker for MET inhibitor therapy. A phase I study of crizotinib and dacomitinib in the same patient population did not

\begin{tabular}{|c|c|c|c|c|c|c|}
\hline \multirow[b]{2}{*}{ Adverse Event Preferred Term } & \multicolumn{2}{|c|}{ Phase $\mathrm{lb}(\mathrm{n}=61)$} & \multicolumn{2}{|c|}{ Phase II $(n=100)$} & \multicolumn{2}{|c|}{ All Patients $(\mathrm{N}=161)$} \\
\hline & All Grades & Grades 3/4 & All Grades & Grades 3/4 & All Grades & Grades 3/4 \\
\hline Total & $61(100)$ & $35(57)$ & 98 (98) & $56(56)$ & 159 (99) & $91(57)$ \\
\hline Nausea & $25(41)$ & $2(3)$ & 33 (33) & $5(5)$ & $58(36)$ & $7(4)$ \\
\hline Decreased appetite & $21(34)$ & $1(2)$ & $31(31)$ & $3(3)$ & $52(32)$ & $4(2)$ \\
\hline Peripheral edema & $15(25)$ & $1(2)$ & 34 (34) & $5(5)$ & $49(30)$ & $6(4)$ \\
\hline Hypoalbuminemia & $13(21)$ & 0 & 33 (33) & $1(1)$ & $46(29)$ & $1(1)$ \\
\hline Vomiting & $23(38)$ & $3(5)$ & $21(21)$ & $3(3)$ & $44(27)$ & $6(4)$ \\
\hline Rash & $18(30)$ & $1(2)$ & $21(21)$ & $2(2)$ & $39(24)$ & $3(2)$ \\
\hline Diarrhea & $16(26)$ & $1(2)$ & $22(22)$ & $1(1)$ & $38(24)$ & $2(1)$ \\
\hline Fatigue & $10(16)$ & 0 & $25(25)$ & $6(6)$ & $35(22)$ & $6(4)$ \\
\hline Paronychia & $16(26)$ & 0 & $17(17)$ & $1(1)$ & $33(20)$ & $1(1)$ \\
\hline Cough & $11(18)$ & $1(2)$ & $20(20)$ & 0 & $31(19)$ & $1(1)$ \\
\hline Increased amylase & $11(18)$ & $5(8)$ & $18(18)$ & $6(6)$ & $29(18)$ & $11(7)$ \\
\hline Dyspnea & $17(28)$ & $6(10)$ & $11(11)$ & $3(3)$ & $28(17)$ & $9(6)$ \\
\hline Anemia & $5(8)$ & $2(3)$ & $21(21)$ & $2(2)$ & $26(16)$ & $4(2)$ \\
\hline Increased blood creatinine & $6(10)$ & 0 & $17(17)$ & 0 & $23(14)$ & 0 \\
\hline Constipation & $8(13)$ & 0 & $14(14)$ & 0 & $22(14)$ & 0 \\
\hline Increased ALT & $6(10)$ & $1(2)$ & $15(15)$ & $2(2)$ & $21(13)$ & $3(2)$ \\
\hline Increased lipase & $5(8)$ & $4(7)$ & $15(15)$ & $6(6)$ & $20(12)$ & $10(6)$ \\
\hline Increased AST & $4(7)$ & $1(2)$ & $15(15)$ & $2(2)$ & $19(12)$ & $3(2)$ \\
\hline Dizziness & $9(15)$ & $2(3)$ & $9(9)$ & $1(1)$ & $18(11)$ & $3(2)$ \\
\hline Increased blood bilirubin & $5(8)$ & 0 & $12(12)$ & $1(1)$ & $17(11)$ & $1(1)$ \\
\hline Hemoptysis & $7(11)$ & 0 & $10(10)$ & $1(1)$ & $17(11)$ & $1(1)$ \\
\hline Insomnia & $8(13)$ & 0 & $9(9)$ & 0 & $17(11)$ & 0 \\
\hline
\end{tabular}




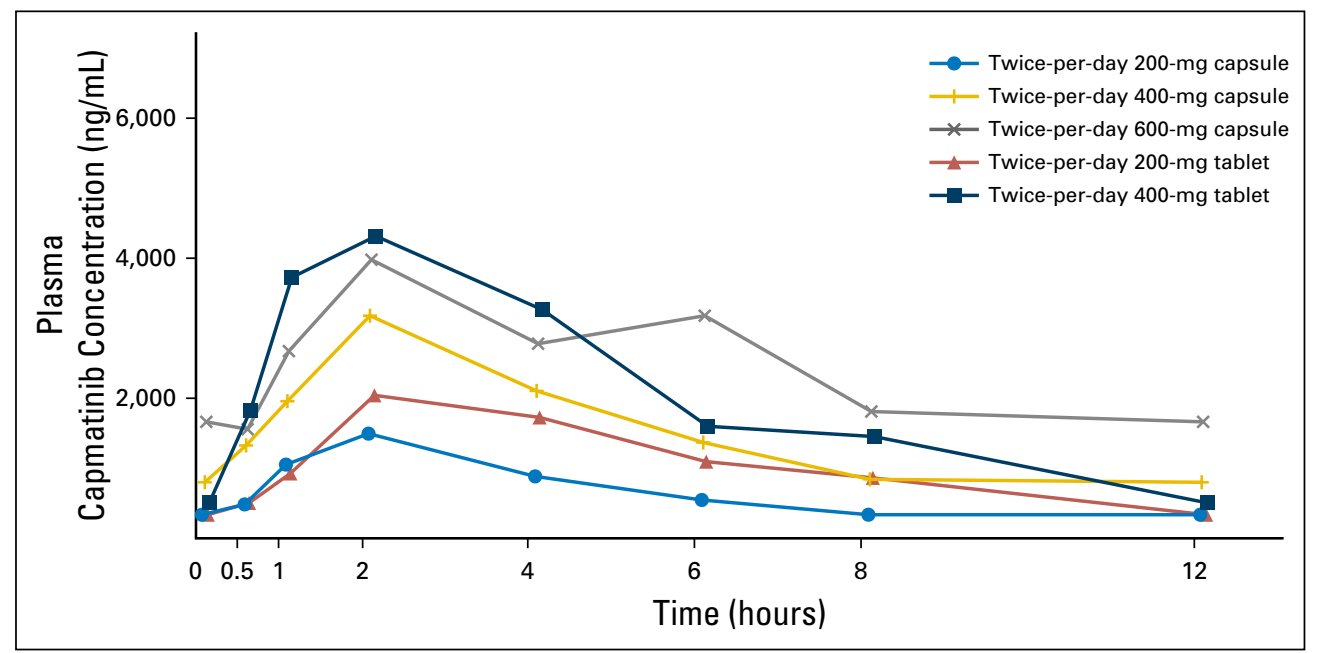

Fig 2. Mean plasma concentration-time curves on cycle 1 day 15 for capmatinib twice-per-day regimens by dose level and formulation (full capmatinib pharmacokinetic analysis set). Zero concentrations at individual timepoints are excluded from geometric mean computation. Concentration at $12 \mathrm{~h}$ after dose is carried forward from the predose value (based on assumption of steady state).

select patients on the basis of MET status and provided limited activity (one partial response; $46 \%$ with stable disease), with no association observed between biomarker expression and clinical activity. $^{23}$ This combination was also associated with unacceptable increased toxicity. ${ }^{23}$ A phase I study of the combination of crizotinib and erlotinib also provided limited activity (ORR, 8\%) in patients with EGFR-mutant NSCLC, albeit in a largely unselected patient population. Furthermore, the MTD of the combination was below the approved dose of either agent, and a phase II study was not initiated. ${ }^{24}$

In contrast to a number of other combination studies, capmatinib in combination with gefitinib is tolerable. The most common study drug-related any-grade and grade 3/4 AEs were nausea and increased lipase or amylase levels, respectively.

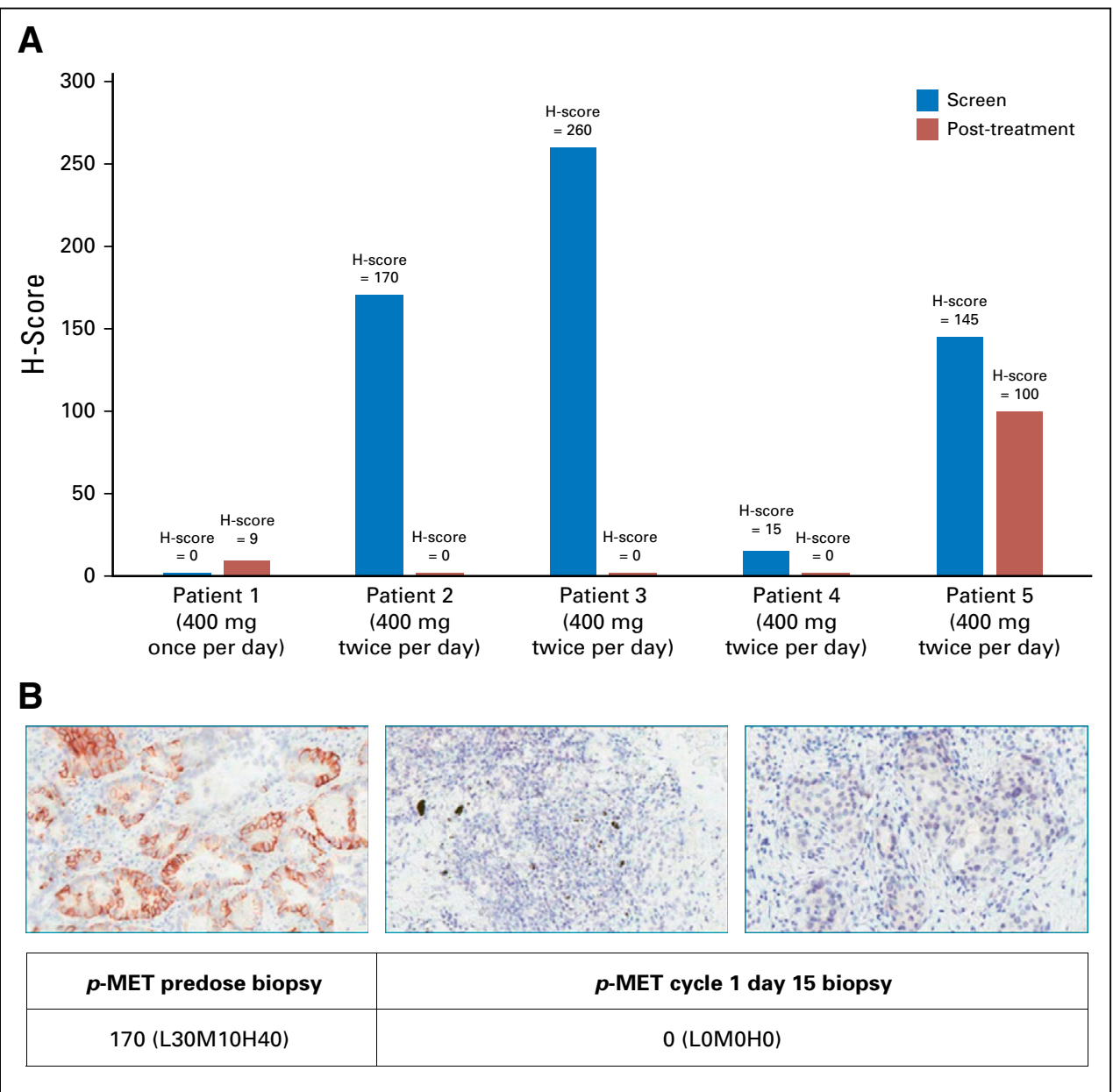

jco.org
Fig 3. (A) Mesenchymal-epithelial transition factor (MET) H-score (phosphorylatedMET [ $p$-MET]) at baseline and post-treatment (cycle 1 day 15). (B) Example pharmacodynamic analysis of $p$-MET in paired predose and postdose (cycle 1 day 15) biopsy immunohistochemistry analysis in one patient treated at the recommended phase II dose of capmatinib $400 \mathrm{mg}$ twice per day plus gefitinib $250 \mathrm{mg}$ once per day, using the $p$-MET cell signaling antibody 3077 . Immunohistochemistry images previously presented (ASCO, 2014) and used with permission of the presenter (Y.-L.W.). 
Peripheral edema believed to be drug related occurred in $22 \%$ of patients; this has been reported for other MET inhibitors ${ }^{25,26}$ and may be a potential drug class effect not specific to capmatinib. Although slightly fewer patients in the capsule group compared with the tablet group experienced AEs (96\% v 100\%) in phase II, there were racial differences between the groups, with the capsule group comprising Chinese patients only, whereas approximately half of the patients in the tablet group were Asian patients from other countries. Overall, capmatinib 400-mg twiceper-day tablets were associated with a tolerability and safety pro-file comparable to that seen with capsules. No significant drug-drug interactions were reported between capmatinib and gefitinib.

On the basis of the PD data from this study, the declared RP2D of capmatinib tablet $400 \mathrm{mg}$ twice per day plus gefitinib $250 \mathrm{mg}$ once per day seemed to be sufficient to completely shut down the MET pathway. Exploratory (five of 161 patients) predose and postdose paired-biopsy analysis of MET phosphorylation by IHC revealed significant MET pathway inhibition after treatment with capmatinib 400-mg twice-per-day capsules in four of the five patients. An association between these markers and clinical outcome, therefore, warrants additional investigation.

This was the first phase II study to focus on the second most predominant resistance mechanism (after T790M mutation) to EGFR-TKI therapy in patients with EGFR-mutated NSCLC, for which limited treatment options are currently available. The combination of capmatinib with gefitinib has been shown to be both feasible and rational, and the data from this study suggest that the combination of capmatinib with an EGFR-TKI may be a promising treatment option for patients with EGFR-mutated, MET-dysregulated NSCLC and particularly for patients with METamplified tumors.

\section{AUTHORS' DISCLOSURES OF POTENTIAL CONFLICTS OF INTEREST}

Disclosures provided by the authors are available with this article at jco.org.

\section{AUTHOR CONTRIBUTIONS}

Conception and design: Yi-Long Wu, James Chih-Hsin Yang, Johan F. Vansteenkiste, Bin Peng, Mikhail Akimov, Daniel S.W. Tan

Administrative support: Mikhail Akimov

Provision of study materials or patients: Yi-Long Wu, Dong-Wan Kim, James Chih-Hsin Yang, Johan F. Vansteenkiste, Daniel S.W. Tan

Collection and assembly of data: Yi-Long Wu, Li Zhang, Dong-Wan Kim, Xiaoqing Liu, Dae Ho Lee, James Chih-Hsin Yang, Johan F. Vansteenkiste, Wu-Chou Su, Daniel S.W. Tan

Data analysis and interpretation: Yi-Long Wu, Li Zhang, Dong-Wan Kim, Dae Ho Lee, James Chih-Hsin Yang, Myung-Ju Ahn, Johan F. Vansteenkiste, Enriqueta Felip, Vincent Chia, Sabine Glaser, Philippe Pultar, Sylvia Zhao, Mikhail Akimov, Daniel S.W. Tan

Manuscript writing: All authors

Final approval of manuscript: All authors

Accountable for all aspects of the work: All authors

\section{REFERENCES}

1. Kobayashi S, Boggon TJ, Dayaram $T$, et al: EGFR mutation and resistance of non-small-cell lung cancer to gefitinib. N Engl J Med 352:786-792, 2005

2. Engelman JA, Zejnullahu K, Mitsudomi T, et al: MET amplification leads to gefitinib resistance in lung cancer by activating ERBB3 signaling. Science 316: 1039-1043, 2007

3. Bean J, Brennan C, Shih JY, et al: MET amplification occurs with or without T790M mutations in EGFR mutant lung tumors with acquired resistance to gefitinib or erlotinib. Proc Natl Acad Sci USA 104: 20932-20937, 2007

4. Chen HJ, Mok TS, Chen ZH, et al: Clinicopathologic and molecular features of epidermal growth factor receptor T790M mutation and c-MET amplification in tyrosine kinase inhibitor-resistant Chinese non-small cell lung cancer. Pathol Oncol Res 15:651-658, 2009

5. Sequist LV, Waltman BA, Dias-Santagata $D$, et al: Genotypic and histological evolution of lung cancers acquiring resistance to EGFR inhibitors. Sci Transl Med 3:75ra26, 2011

6. Yu HA, Arcila ME, Rekhtman N, et al: Analysis of tumor specimens at the time of acquired resistance to EGFR-TKI therapy in 155 patients with EGFR-mutant lung cancers. Clin Cancer Res 19:2240-2247, 2013

7. Minari R, Bordi $P$, Tiseo M: Third-generation epidermal growth factor receptor-tyrosine kinase inhibitors in T790M-positive non-small cell lung cancer: Review on emerged mechanisms of resistance. Transl Lung Cancer Res 5:695-708, 2016

8. Liu $X$, Wang $Q$, Yang G, et al: A novel kinase inhibitor, INCB28060, blocks c-MET-dependent signaling, neoplastic activities, and cross-talk with EGFR and HER-3. Clin Cancer Res 17:7127-7138, 2011

9. Wu Y, Yang JC, Kim D, et al: Safety and efficacy of INC280 in combination with gefitinib in patients with EGFR-mutated, MET-positive NSCLC: A single-arm phase Ib/ll study. J Clin Oncol 32, 2014 (suppl; abstr 8017)

10. Frampton GM, Ali SM, Rosenzweig M, et al: Activation of MET via diverse exon 14 splicing alterations occurs in multiple tumor types and confers clinical sensitivity to MET inhibitors. Cancer Discov 5 : 850-859, 2015

11. Lara MS, Holland WS, Chinn D, et al: Preclinical evaluation of MET inhibitor INC-280 with or without the epidermal growth factor receptor inhibitor erlotinib in nonsmall-cell lung cancer. Clin Lung Cancer 18:281-285, 2017

12. Neuenschwander $B$, Branson M, Gsponer $T$ : Critical aspects of the Bayesian approach to phase I cancer trials. Stat Med 27:2420-2439, 2008

13. Jackman D, Pao W, Riely GJ, et al: Clinical definition of acquired resistance to epidermal growth factor receptor tyrosine kinase inhibitors in non-smallcell lung cancer. J Clin Oncol 28:357-360, 2010

14. Scheffler M, Di Gion P, Doroshyenko O, et al: Clinical pharmacokinetics of tyrosine kinase inhibitors: Focus on 4-anilinoquinazolines. Clin Pharmacokinet 50:371-403, 2011

15. Tsuta K, Kozu Y, Mimae T, et al: c-MET/ phospho-MET protein expression and MET gene copy number in non-small cell lung carcinomas. J Thorac Oncol 7:331-339, 2012

16. Onitsuka $T$, Uramoto $H$, Ono $K$, et al: Comprehensive molecular analyses of lung adenocarcinoma with regard to the epidermal growth factor receptor, $\mathrm{K}$-ras, MET, and hepatocyte growth factor status. J Thorac Oncol 5:591-596, 2010
17. Kawakami H, Okamoto I, Okamoto W, et al: Targeting MET amplification as a new oncogenic driver. Cancers (Basel) 6:1540-1552, 2014

18. Spigel DR, Ervin TJ, Ramlau RA, et al: Randomized phase II trial of onartuzumab in combination with erlotinib in patients with advanced non-small-cell lung cancer. J Clin Oncol 31:4105-4114, 2013

19. Spigel DR, Edelman MJ, O'Byrne KJ, et al: Onartuzumab plus erlotinib versus erlotinib in previously treated stage IIIb or IV NSCLC: Results from the pivotal phase III randomized, multicenter, placebocontrolled METLung (OAM4971g) global trial. J Clin Oncol 32, 2014 (suppl; abstr 8000)

20. Yoshioka H, Azuma K, Yamamoto N, et al: A randomized, double-blind, placebo-controlled, phase III trial of erlotinib with or without a c-Met inhibitor tivantinib (ARO 197) in Asian patients with previously treated stage IIIB/IV nonsquamous nonsmall-cell lung cancer harboring wild-type epidermal growth factor receptor (ATTENTION study). Ann Oncol 26:2066-2072, 2015

21. Calles A, Kwiatkowski N, Cammarata BK, et al: Tivantinib (ARQ 197) efficacy is independent of MET inhibition in non-small-cell lung cancer cell lines. Mol Oncol 9:260-269, 2015

22. $W u$ YL, Soo RA, Kim D-W, et al: Tolerability, efficacy and recommended phase II dose (RP2D) of tepotinib plus gefitinib in Asian patients with c-Metpositive/EGFRmutant NSCLC: phase Ib data. J Clin Oncol 34, 2016 (suppl; abstr e20501)

23. Jänne PA, Shaw AT, Camidge DR, et al: Combined pan-HER and ALK/ROS1/MET inhibition with dacomitinib and crizotinib in advanced non-small cell lung cancer: Results of a phase I study. J Thorac Oncol 11:737-747, 2016 
24. Ou SI, Govindan R, Eaton KD, et al: Phase I results from a study of crizotinib in combination with erlotinib in patients with advanced nonsquamous non-small cell lung cancer. J Thorac Oncol 12: 145-151, 2017
25. Falchook GS, Kurzrock R, Amin HM, et al: Efficacy, safety, biomarkers, and Phase II dose modeling in a phase I trial of the oral selective c-MET inhibitor tepotinib (MSC2156119J). J Clin Oncol 33, 2015 (suppl; abstr 2591)
26. Salgia R, Patel $P$, Bothos J, et al: Phase I doseescalation study of onartuzumab as a single agent and in combination with bevacizumab in patients with advanced solid malignancies. Clin Cancer Res 20: 1666-1675, 2014

\section{Affiliations}

Yi-Long Wu, Guangdong General Hospital and Guangdong Academy of Medical Sciences; Li Zhang, Sun Yat-sen University Cancer Center, Guangdong; Xiaoqing Liu, Affiliated Hospital of the Chinese Academy of Military Medical Sciences, Beijing; Sylvia Zhao and Bin Peng, Novartis Institutes for Biomedical Research, Shanghai, People's Republic of China; Dong-Wan Kim, Seoul National University Hospital; Dae Ho Lee, University of Ulsan College of Medicine; Myung-Ju Ahn, Samsung Medical Center, Seoul, Republic of Korea; James Chih-Hsin Yang, National Taiwan University Hospital, Taipei; Wu-Chou Su, National Cheng Kung University Hospital, Tainan, Taiwan; Johan F. Vansteenkiste, University Hospital KU Leuven, Leuven, Belgium; Enriqueta Felip, Vall d'Hebron University Hospital, Barcelona, Spain; Vincent Chia and Philippe Pultar, Novartis Pharmaceuticals, East Hanover, NJ; Sabine Glaser and Mikhail Akimov, Novartis Pharma AG, Basel, Switzerland; and Daniel S.W. Tan, National Cancer Centre Singapore, Singapore.

Supported by Novartis Pharma AG.

\section{Support}

\section{Prior Presentation}

Presented in part at the 2016 ASCO Annual Meeting, Chicago, IL, June 3-7, 2016. 
Phase Ib/II Study of Capmatinib (INC280) Plus Gefitinib After Failure of Epidermal Growth Factor Receptor (EGFR) Inhibitor Therapy in Patients With EGFR-Mutated, MET Factor-Dysregulated Non-Small-Cell Lung Cancer

The following represents disclosure information provided by authors of this manuscript. All relationships are considered compensated. Relationships are self-held unless noted. I = Immediate Family Member, Inst = My Institution. Relationships may not relate to the subject matter of this manuscript. For more information about ASCO's conflict of interest policy, please refer to www.asco.org/rwc or ascopubs.org/jco/site/ifc.

\section{Yi-Long Wu}

Honoraria: AstraZeneca, Eli Lilly, Roche, Pfizer, Sanofi, Boehringer Ingelheim

Consulting or Advisory Role: AstraZeneca, Roche, Merck, Boehringer Ingelheim

Research Funding: Boehringer Ingelheim (Inst), Roche (Inst)

Li Zhang

Consulting or Advisory Role: Boehringer Ingelheim

Research Funding: BMS (Inst)

\section{Dong-Wan Kim}

No relationship to disclose

\section{Xiaoqing Liu}

No relationship to disclose

\section{Dae Ho Lee}

Honoraria: AstraZeneca, Boehringer Ingelheim, Bristol-Myers Squibb, CJ Healthcare, Eli Lilly, Janssen, Merck, Merck Sharp \& Dohme,

Mundipharma, Novartis, Ono Pharmaceutical, Pfizer, Roche, Samyang, ST Cube

Consulting or Advisory Role: Korean Ministry of Food and Drug Safety, Korean Health Insurance Review and Assessment Service, Korean National Evidence-Based Collaborating Agency, Korean National Cancer Control Planning Board

James Chih-Hsin Yang

Honoraria: Boehringer Ingelheim, Roche, Chugai Pharma, MSD, AstraZeneca, Novartis

Consulting or Advisory Role: Boehringer Ingelheim, Novartis,

AstraZeneca, Roche/Genentech, Eli Lilly, MSD Oncology, Merck Serono, Celgene, Pfizer, Ono Pharmaceutical, Bristol-Myers Squibb, AstraZeneca, Yuhan, Hansoh, Takeda, Daiichi Sankyo

\section{Myung-Ju Ahn}

No relationship to disclose

Johan F. Vansteenkiste

Honoraria: Bristol-Myers Squibb, AstraZeneca, MSD Oncology,

Consulting or Advisory Role: Novartis (Inst), Boehringer Ingelheim (Inst), AstraZeneca (Inst), MSD Oncology (Inst), Bristol-Myers Squibb (Inst), Merck Serono (Inst)

Research Funding: MSD (Inst)

\section{Wu-Chou Su}

Travel, Accommodations, Expenses: Chugai Pharma, Merck, Ono Pharmaceutical

\section{Enriqueta Felip}

Consulting or Advisory Role: Astra Zeneca, Boehringer Ingelheim, Eli Lilly, Guardant Health, Merck Sharp \& Dohme, Novartis, Pfizer, Roche, Takeda, Merck, Bristol-Myers Squibb

Speakers' Bureau: AstraZeneca, Boehringer Ingelheim, Bristol-Myers Squibb, Eli Lilly, Guardant Health, Merck Sharp \& Dohme, Novartis, Pfizer, Roche, Takeda

\section{Vincent Chia \\ Employment: Novartis}

\section{Sabine Glaser}

Employment: Novartis Pharma

Stock or Other Ownership: Novartis Pharma AG

\section{Philippe Pultar}

Employment: Novartis

Stock or Other Ownership: Novartis

\section{Sylvia Zhao}

Employment: Novartis

Stock or Other Ownership: Novartis

\section{Bin Peng}

Employment: Novartis

Stock or Other Ownership: Novartis

Mikhail Akimov

Employment: Novartis Pharma AG

Stock or Other Ownership: Novartis Pharma AG

Daniel S.W. Tan

Honoraria: Novartis, Merck, Boehringer Ingelheim, Roche Consulting or Advisory Role: Novartis, Pfizer, AstraZeneca Research Funding: Novartis, GlaxoSmithKline, AstraZeneca 


\section{Acknowledgment}

Editorial support for this article was provided by Matthew Naylor and funded by Novartis Pharmaceuticals.

\section{Appendix}

\section{Treatment Plan and Drug Administration}

Patients were dosed continuously in 28-day cycles, and treatment continued until disease progression (Response Evaluation Criteria in Solid Tumors [RECIST] version 1.1; investigator assessed), unacceptable toxicity precluding additional treatment, pregnancy, discontinuation at the discretion of the investigator or patient, withdrawal of consent, loss to follow-up, or death. Tumor lesions were assessed (RECIST version 1.1; investigator confirmed) using computed tomography unless contraindicated, in which case magnetic resonance imaging with contrast was performed. Efficacy imaging assessments were carried out at screening, every two cycles, 4 weeks after any reported response, and at the end of treatment (if no scan $\leq 30$ days before the end of treatment). Safety assessments were performed on the basis of all adverse events assessed according to Common Terminology Criteria for Adverse Events version 4.0, clinical laboratory data, and physical examinations.

\section{Statistical Analysis of the Primary Objective (phase II)}

For the primary analysis, the overall response rate (ORR) was estimated using a Bayesian approach with a minimally informative beta prior distribution. However, the ORR estimate on the basis of the frequency of complete or partial responses is presented; results were almost identical.

On the basis of the posterior distribution, the probability that the true ORR lies in the following categories was calculated: unacceptable efficacy: $0 \%, 5 \%$; limited efficacy: $5 \%, 20 \%$; clinically relevant efficacy, $20 \%, 100 \%$. The criterion for evidence of clinically relevant efficacy of the combination was an observed ORR of $\geq 20 \%$ and a posterior risk of $<2.5 \%$ that the true ORR was in the unacceptable efficacy category.

\section{Progression-Free Survival}

In 100 evaluable patients treated at the recommended phase II dose (mesenchymal-epithelial transition factor [MET] positivity initially defined as MET gene copy number [GCN] $\geq 5$ or $50 \%$ of tumor cells with immunohistochemistry [IHC] $2+/ 3+$, revised to $50 \%$ of tumor cells with IHC $3+$ or IHC $2+$ plus MET GCN $\geq 5$, and then to $50 \%$ of tumor cells with IHC $3+$ or MET GCN $\geq 4$ ), the median progression-free survival (PFS; secondary end point) for all patients was 5.5 months (95\% CI, 3.8 to 5.6 months). Median PFS in the GCN $\geq 6(\mathrm{n}=36), 4 \leq \mathrm{GCN}<6(\mathrm{n}=18)$, and GCN $<4(\mathrm{n}=41)$ subgroups was 5.49 months $(95 \%$ CI, 4.21 to 7.29 months), 5.39 months ( $95 \%$ CI, 3.65 to 7.46 months), and 3.91 months (95\% CI, 3.65 to 5.55 months), respectively (Appendix Fig A2A, online only). Median PFS in the IHC $3+(\mathrm{n}=78)$ and IHC $2+/ \mathrm{GCN} \geq 5(\mathrm{n}=8)$ subgroups was 5.45 months $(95 \% \mathrm{CI}, 3.71$ to 7.10 months) and 7.29 months (95\% CI, 1.81 to 9.07 months), respectively (Appendix Fig A2B). 


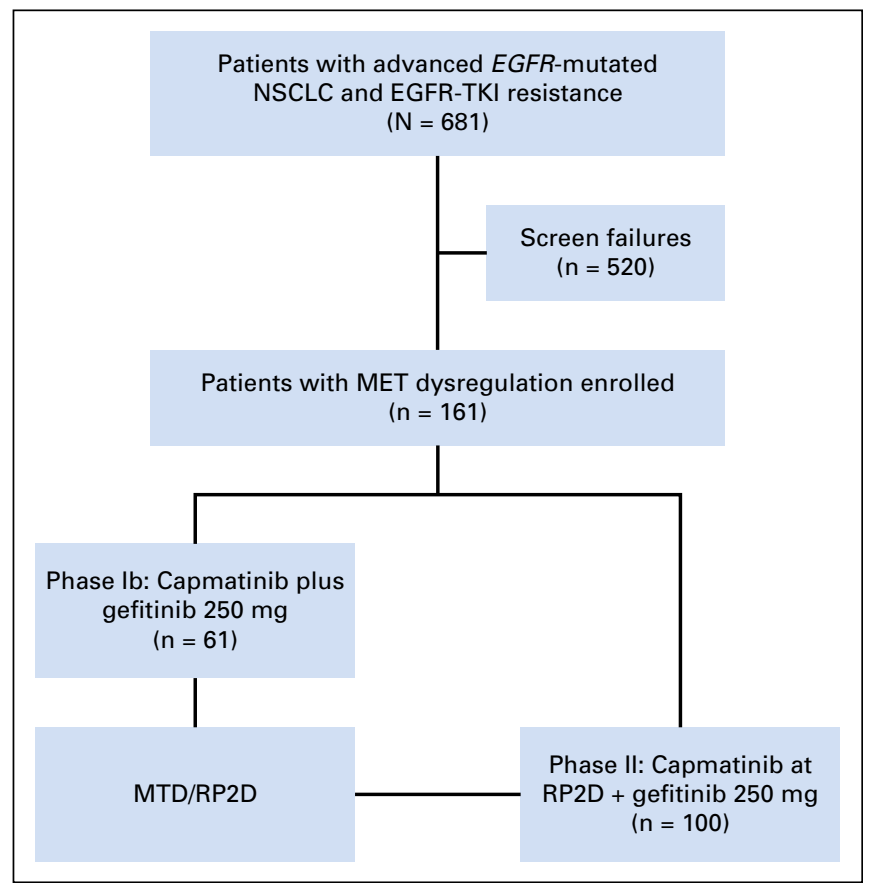

Fig A1. CONSORT diagram. MET, mesenchymal-epithelial transition factor; MTD, maximum tolerated dose; NSCLC, non-small-cell lung cancer; RP2D, recommended phase II dose; TKI, tyrosine kinase inhibitor. 


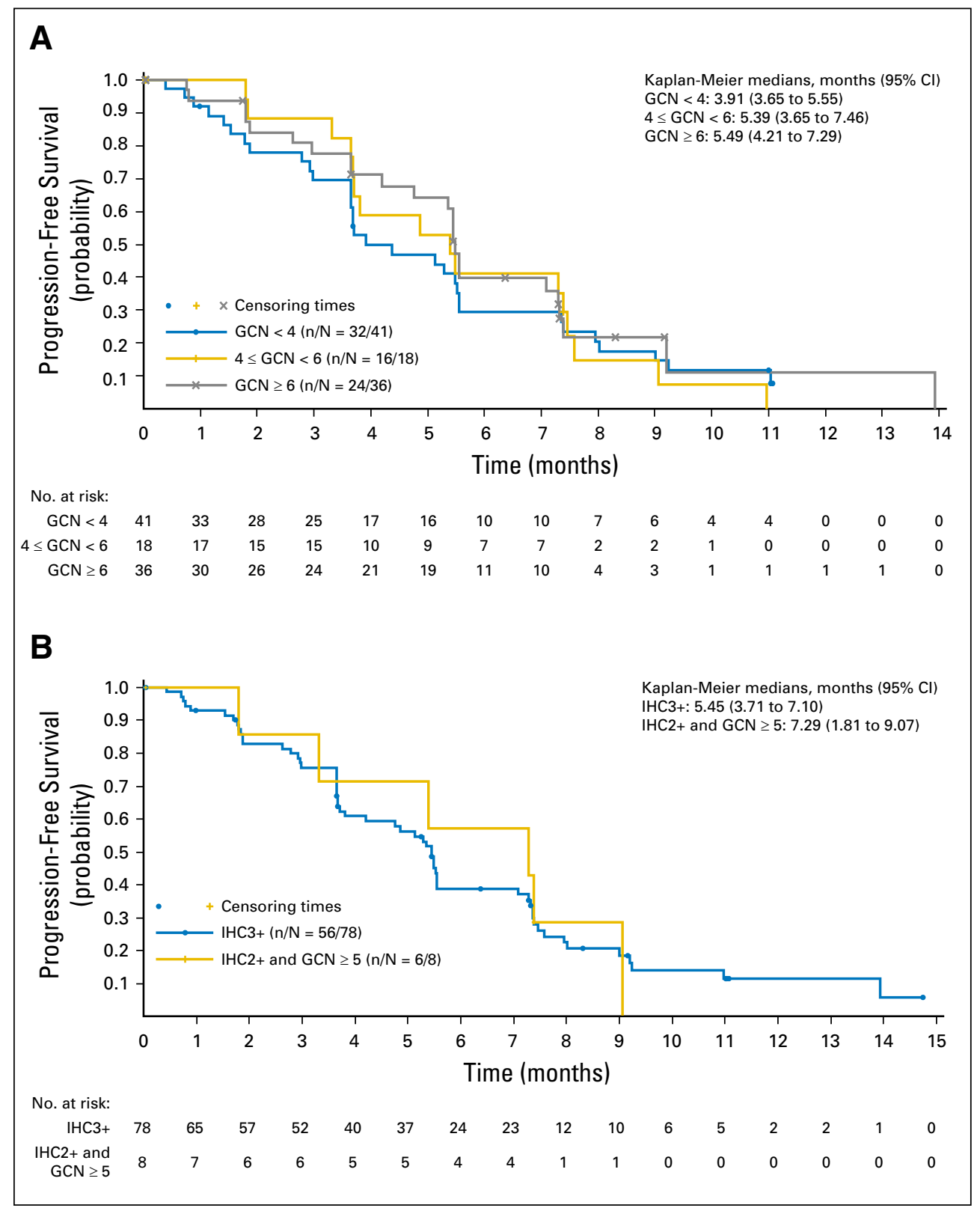

Fig A2. (A) Kaplan-Meier plot of progression-free survival for patients treated in the phase Il part by gene copy number (GCN) subgroup (full analysis set). (B) Kaplan-Meier plot of progression-free survival for patients treated in the phase II part by GCN subgroup (full analysis set). IHC, immunohistochemistry. 


\begin{tabular}{|c|c|c|c|c|c|}
\hline IHC Score & $\mathrm{GCN}<4$ & $4 \leq \mathrm{GCN}<6$ & $\mathrm{GCN} \geq 6$ & Missing/Unknown & Total \\
\hline 0 & 0 & $2(2)$ & $2(2)$ & 0 & $4(4)$ \\
\hline $1+$ & $2(2)$ & 0 & 0 & 0 & $2(2)$ \\
\hline $2+$ & $8(8)$ & $6(6)$ & $2(2)$ & 0 & $16(16)$ \\
\hline $3+$ & $31(31)$ & $10(10)$ & 32 (32) & $5(5)$ & $78(78)$ \\
\hline Missing/unknown & 0 & 0 & 0 & 0 & 0 \\
\hline Total & $41(41)$ & $18(18)$ & $36(36)$ & $5(5)$ & $100(100)$ \\
\hline
\end{tabular}

\begin{tabular}{|c|c|c|c|c|c|c|c|c|c|}
\hline \multirow[b]{2}{*}{ PK Parameter } & \multicolumn{4}{|c|}{ Once per Day } & \multicolumn{5}{|c|}{ Twice per Day } \\
\hline & $\begin{array}{c}100-m g \\
\text { Cap (n = 4) }\end{array}$ & $\begin{array}{c}200-m g \\
\text { Cap (n = 7) }\end{array}$ & $\begin{array}{c}400-m g \\
\text { Cap (n =6) }\end{array}$ & $\begin{array}{c}800-m g \\
\text { Cap (n = 4) }\end{array}$ & $\begin{array}{c}200-m g \\
\text { Cap (n = 4) }\end{array}$ & $\begin{array}{c}400-\mathrm{mg} \\
\text { Cap }(\mathrm{n}=10)\end{array}$ & $\begin{array}{c}600-m g \\
\text { Cap }(n=2)\end{array}$ & $\begin{array}{c}200-m g ~ T a b \\
(n=7)\end{array}$ & $\begin{array}{c}\text { 400-mg Tab } \\
(\mathrm{n}=7)\end{array}$ \\
\hline $\mathrm{AUC}_{0-12 \mathrm{~h}}, \mathrm{ng}^{*} \mathrm{~h} / \mathrm{mL}$ & $\mathrm{n}=4$ & $n=7$ & $\mathrm{n}=5$ & $\mathrm{n}=3$ & $n=4$ & $\mathrm{n}=10$ & $\mathrm{n}=2$ & $n=7$ & $\mathrm{n}=7$ \\
\hline Geomean & 4,130 & 7,830 & 26,900 & 23,600 & 8,420 & 19,400 & 34,800 & 13,400 & 28,200 \\
\hline CV\% & 54.6 & 65.4 & 50.0 & 126.6 & 66.9 & 57.3 & 56.8 & 29.4 & 20.6 \\
\hline $\mathrm{C}_{\max }, \mathrm{ng} / \mathrm{mL}$ & $n=4$ & $n=7$ & $n=6$ & $\mathrm{n}=4$ & $n=4$ & $n=10$ & $n=2$ & $n=7$ & $n=7$ \\
\hline Geomean & 701 & 1,020 & 3,150 & 4,800 & 1,740 & 3,740 & 4,630 & 2,470 & 6,560 \\
\hline CV\% & 75.6 & 121.3 & 188.4 & 149.9 & 63.1 & 57.6 & 44.3 & 27.2 & 27.0 \\
\hline$T_{\max }$ (hours) & $n=4$ & $n=7$ & $n=6$ & $n=4$ & $n=4$ & $n=10$ & $n=2$ & $n=7$ & $n=7$ \\
\hline Median & 1.96 & 2.00 & 2.00 & 2.05 & 1.50 & 2.00 & 5.00 & 2.00 & 1.08 \\
\hline Range & $1.50-3.92$ & $1.00-24.0$ & $1.98-6.00$ & $1.92-5.97$ & $1.00-3.98$ & $0.50-4.00$ & $4.00-6.00$ & $1.00-4.00$ & $1.00-4.00$ \\
\hline $\mathrm{T}_{1 / 2}$ (hours) & $\mathrm{n}=4$ & $\mathrm{n}=6$ & $n=5$ & $n=3$ & $n=3$ & $n=9$ & - & $\mathrm{n}=7$ & $n=6$ \\
\hline Geomean & 3.83 & 4.82 & 3.14 & 3.60 & 3.09 & 2.81 & - & 3.37 & 3.08 \\
\hline CV\% & 14.5 & 37.1 & 11.4 & 23.6 & 33.3 & 38.6 & - & 52.5 & 27.3 \\
\hline \multicolumn{10}{|c|}{ 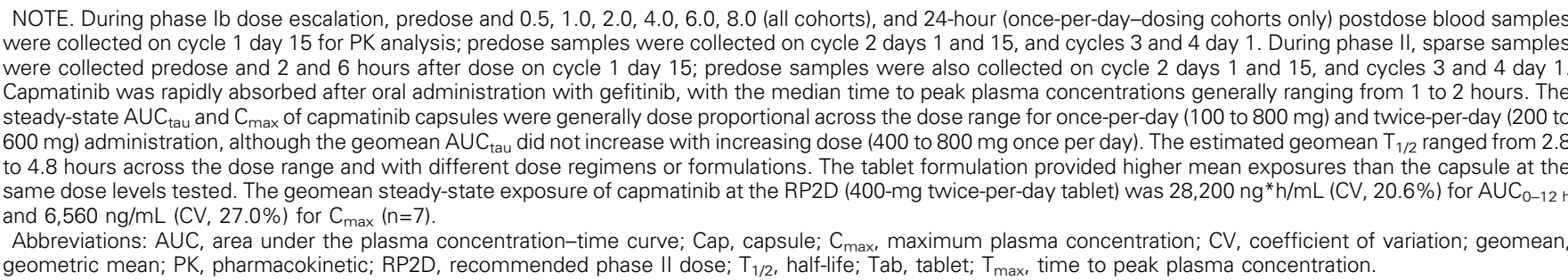 } \\
\hline
\end{tabular}

